\title{
Revisión de los patrones de distribución de Crocodylus niloticus Laurenti 1768, en Etiopía, y estima de tamaño corporal de grandes ejemplares del lago Chamo
}

\section{Review of the distribution patterns of Crocodylus ni- loticus Laurenti 1768, in Ethiopia, and estimation of the body size of large specimens from Lake Chamo}

Conrado Tejado-Lanseros ${ }^{1 *}$

(6)

\section{Resumen}

Se ha elaborado un análisis actualizado de los patrones de distribución de Crocodylus niloticus Laurenti, 1768 en Etiopía. Se determina la presencia de la especie en 17 cauces fluviales: ríos Mareb, Tekeze, Abbai, Awash, Gibe, Gojebe, Omo, Baro, Aluoro, Ghilo, Akobo, Hare, Kulfo, Sagan, Daua, Genale y Webi Shebeli y 12 lagos: Abe, Yardi, Beda, Beseka, Koka, Ziway, Langano, Abaya, Chamo, Chew Bahir, Turkana y Murle. La estimación de tamaño corporal de grandes ejemplares procedentes del lago Chamo, en función de muestras craneales, ofrece resultados de tres individuos que superan los 5 metros de longitud total ( $L C T=5,11 \mathrm{~m}, 5,26 \mathrm{~m}$ y 5,35 $\mathrm{m}$ ) y dos individuos que se acercan a los 5 metros ( $(\mathrm{LCT}=4,84 \mathrm{~m}$ y 4,99 m). Por ello se destaca la población de C. niloticus del lago Chamo como una de las más relevantes del continente africano.

Palabras clave: tamaño corporal, Crocodylus niloticus, distribución, Etiopía, lago Chamo.

\section{Abstract}

An up-to-date analysis of the distribution patterns of Crocodylus niloticus Laurenti, 1768, in Ethiopia has been carried out. The presence of this species in 17 water courses has been determined: Mareb, Tekeze, Abbai, Awash, Gibe, Gojebe, Omo, Baro, Aluoro, Ghilo, Akobo, Hare,

\footnotetext{
1 Instituto Alavés de la Naturaleza. Departamento de Zoología-Vertebrados.

E-48014 • Apdo. $2092 \cdot 01008$ Vitoria-Gasteiz.

* info@ian-ani.org • Correspondencia: conradotejado@gmail.com
} 
Kulfo, Sagan, Daua, Genale and Webi Shebeli, as well as 12 lakes: Abe, Yardi, Beda, Beseka, Koka, Ziway, Langano, Abaya, Chamo, Chew Bahir, Turkana and Murle. Estimations of body size in large individuals from lake Chamo, based on cranial samples, have revealed three individuals of over 5 metres in total length ( $L C T=5,11 \mathrm{~m}, 5,26 \mathrm{~m}$ and 5,35 m) and two individuals of just under 5 metres ( $L C T=4,84 \mathrm{~m}$ and 4,99 $\mathrm{m}$ ). The article highlights the importance of the population of C. niloticus in lake Chamo as one of the most relevant on the African continent.

Key words: body size, Crocodylus niloticus, distribution, Ethiopia, lake Chamo.

\section{Laburpena}

Aktualizazioa gauzatu da Crocodylus niloticus Laurenti, 1768 espezieak Etiopian duen banaketaren patroiena. 17 ibilgutan espeziearen presentzia baieztatu da: Mareb erreka, Tekeze, Abbai, Awash, Gibe, Gojebe, Omo, Baro, Aluoro, Ghilo, Akobo, Hare, Kulfo, Sagan, Daua, Genale eta Webi Shebeli eta 12 laku: Abe, Yardi, Beda, Beseka, Koka, Ziway, Langano, Abaya, Chamo, Chew Bahir, Turkana eta Murle. Chamo lakutik jasotako banako handien gorputz tamainaren estimazioek erakusten dute, garezur laginak oinarri hartuta, guztira 5 metroko luzera gainditzen duten hiru indibiduo daudela ( $L C T=5,11 \mathrm{~m}, 5,26 \mathrm{~m}$ y 5,35 $\mathrm{m}$ ) eta beste bi 5 metroko luzerara hurbiltzen direla ( $L C T=4,84$ m y 4,99 m). Horregatik nabarmentzekoa da Chamo lakuko C. niloticus espeziearen populazioa, Afrika kontinenteko aipagarrienetako bat.

Gako hitzak: gorputz tamaina, Crocodylus niloticus, banaketa, Etiopia, Chamo lakua.

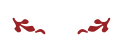

\section{Introducción}

El cocodrilo del Nilo, Crocodylus niloticus Laurenti, 1768, es la especie de crocodiliano que presenta más amplia distribución dentro del continente africano, ocupando en mayor o menor grado la práctica totalidad de los países localizados al sur del Sahara (Ferguson, 2010). El volumen de estudios dedicado al conocimiento de su estatus y distribución varía considerablemente entre regiones, siendo aceptable el obtenido para las poblaciones de África Austral y Oriental. Por el contrario, la información disponible para un amplio rango de países, principalmente del África Central y Occidental, es insuficiente o prácticamente inexistente atendiendo al banco de datos recientemente establecido para la especie por UNEP - WCMC (United Nations Environment Programe's World Conservation Monitoring Centre) y por el "Crocodile Specialist Group" (Lainez, 2009).

Los estudios científicos realizados en territorio etíope históricamente han sido puntuales y fragmentados (Andeberhan, 1977; Bolton, 1973; Chapman, 1973), considerándose el estatus de la especie en el país hasta la década de los ochenta del siglo pasado como 
"Desconocida" (IUCN, 1982). En décadas anteriores se obtuvieron referencias geográficamente muy localizadas en países vecinos como Kenia, que comparte grandes lagos con Etiopía, como el Turkana (Graham, 1968), así como el atisbo de los primeros análisis de conjunto de la especie en informes que no fueron publicados (Chapman, no publ.). A comienzos de 1973 la Comisión de Ecología de la IUCN hace público un informe global para C. niloticus en África, con un capítulo específico para Etiopía (IUCN, 1973). Posteriormente, investigadores integrados en el Crocodile Specialist Group publican notas sobre el área de estudio (Grebe \& Wakjira, 1996; Grebe, 2002). Solo a partir de 2007 e impulsado por el interés que ha suscitado la gran población de C. niloticus asentada en el lago Chamo, el gobierno etíope autorizó el desarrollo de investigaciones pormenorizadas en este espacio lacustre (Whitaker \& Whitaker, 2007), que recientemente se han complementado con el estudio de un plan de aprovechamiento sostenible en poblaciones sometidas a explotación cinegética (Shirley et al., 2014). En 1971 la especie se había enrarecido en el conjunto del territorio: consecuentemente, en 1972 se prohibió la caza comercial. Etiopía está adherida a la Convención Internacional de Tráfico de Especies Amenazadas (CITES), que regula la comercialización de productos obtenidos del cocodrilo. En 1988 la especie fue transferida del Apéndice I, con el máximo rango de protección, al Apéndice II, que autoriza una cuota de exportación anual de pieles procedentes de ejemplares criados en cautividad.

La comparativa entre algunos estudios recientes (Tefera, 2014) y publicaciones con la autoría de especialistas consolidados (Largen \& Spawls 2010), ofrece resultados localmente contradictorios en lo referente al rango de distribución de C. niloticus en Etiopía. Ello justifica la conveniencia de un mayor aporte de información fiable de carácter corológico para el conjunto de las regiones del país, y especialmente para determinados lagos del Valle del Rift (Ziway, Awasa y Langano).

Se ha planteado la existencia de una segunda especie de cocodrilo en territorio etíope (Crocodylus suchus), con importantes implicaciones a nivel de conservación. Estudios moleculares recientes indican que las poblaciones de África Occidental presentan un alto grado de divergencia respecto a las del resto del continente (Hekkala et al., 2011). Crocodylus suchus se ha reconocido y catalogado como tal especie en la Lista roja de IUCN, habiéndose clasificado como "Vulnerable". Posiblemente se catalogará como "En Peligro" en un futuro próximo. Registros contemporáneos para esta nueva especie existen en países limítrofes como Uganda (Valle de Kidepo) y Sudán del Sur (Hekkala et al., 2011). Aún no ha sido evaluada su presencia potencial en Etiopía, pero ejemplares fotografiados en el río Awash presentan caracteres morfológicos propios de C. suchus (Shirley et al., 2014). En el presente artículo se hace referencia únicamente a C. niloticus para el conjunto del territorio, a la espera del desarrollo de investigaciones de naturaleza genética y taxonómica que determinen con seguridad la presencia de ambas especies.

El objetivo del presente estudio fue ofrecer información de carácter corológico valiosa a nivel comparativo para un espacio geográfico muy amplio y difícil de muestrear, por la carencia de infraestructuras de acceso y lo agreste del medio. Igualmente se aporta infor- 
mación sobre el tamaño corporal de grandes ejemplares procedentes de la población asentada en el lago Chamo, a partir de medidas craneales. La toma de datos biométricos de cráneos es importante ya que éstos, que pueden ser considerados piezas de museo, no se encuentran en las mejores condiciones de conservación, estando inmersos en un proceso de deterioro y pudiendo llegar a desaparecer.

\section{Material y métodos}

El área de estudio engloba el conjunto del territorio etíope, un espacio con notable abundancia de masas fluviales y lacustres. La metodología aplicada para poder cubrir de forma global tan amplio espacio se ha dividido en tres fases. Una primera, de intenso trabajo de documentación bibliográfica, prolongada durante el periodo de muestreo, con la obtención de información complementaria, ofrecida por especialistas del Museo de Ciencias Naturales de Adis Abeba. Una segunda fase tuvo lugar en los meses de octubre y noviembre de 2012, en la que se realizaron prospecciones diurnas y nocturnas, recorriendo un total de $2.870 \mathrm{~km}$ muestreando 7 cauces fluviales y 12 masas lacustres en las regiones de Afar, Awash, Oromiya, Valle del Rift y cuenca del río Omo. Para determinar la presencia de cocodrilos en un cauce fluvial o masa lacustre, durante el día se procedió a la localización de ejemplares en puntos de querencia de termorregulación, básicamente en las orillas en masas de terreno emergente dentro del espacio acuático. Se complementaron prospecciones diurnas con nocturnas para optimizar las garantías de localización, especialmente en aquellos lugares de baja densidad de ejemplares. Para ello se utilizaron canoas y botes con fondo metálico de unos $7 \mathrm{~m}$ de longitud, éstos últimos en aquellos puntos con ejemplares de considerable tamaño, como es el caso de los lagos Chamo y Abaya. El desplazamiento en los lagos se realizó a una distancia aproximada de unos $50 \mathrm{~m}$ de la orilla - variable en función del calado del fondo, las rocas y la vegetación sumergida -, y a una velocidad de 8 - 10 km/hora. Durante el día se utilizaron prismáticos de $10 \times 42 \mathrm{~mm}$ y en los recorridos nocturnos focos de medio alcance, que permiten la detección de los reptiles por el reflejo de la luz en sus ojos (tapetum lucidum). Todo el muestreo se realizó según protocolos estandarizados utilizados para investigaciones de naturaleza demográfica y distributiva (Shirley et al., 2009; Webb \& Smith, 1987). Se utilizó un GPS (Garmin Etrex), y las ubicaciones fueron registradas en cartografía 1:50.000 proporcionada por la Ethiopian Mapping Authority (EMA). En una tercera fase, y de forma simultánea al trabajo de campo, se obtuvo información de primera mano ofrecida por miembros de EWCA, de aquellos puntos que no fueron prospectados. Esta base de datos fue cotejada con la información bibliográfica disponible para completar áreas fronterizas de difícil acceso, o con conflictos tribales latentes que no hacían recomendable su visita (Gambella, Tigray y Somali). El registro de un único ejemplar de C. niloticus en un determinado cauce sirvió para asignar su presencia comprobada (+) en el mismo, independientemente de las densidades observadas. 
En todos los casos se contó con el asesoramiento y compañía de competentes guías locales y personal de Ethiopian Wildlife Conservation Authority (EWCA). Para la estima de longitud corporal total de grandes ejemplares procedentes del lago Chamo, basadas en el tamaño craneal, se aplicó la media aritmética proporcional multiplicada por 7,8, índice establecido previamente en estudios de morfología comparativa por otros especialistas para esta especie en el mismo lago (Whitaker \& Whitaker, 2008).

\section{Resultados}

Con un conjunto de 97 avistamientos de C. niloticus, de los cuáles 20 correspondieron a cauces fluviales y 77 a lagos, se elaboró una base de datos al finalizar las prospecciones. Se ha obtenido la presencia comprobada (+) a partir de observaciones directas en cinco ríos (Awash, Gibe, Gojebe, Omo y Kulfo) y seis lagos (Beseka, Langano, Abaya, Chamo, Turkana y Murle). A partir de registros bibliográficos e información de EWCA se asignó como positiva la presencia de cocodrilo del Nilo en otros 12 ríos (Mareb, Tekeze, Abbai, Baro, Aluoro, Gilo, Akobo, Hare, Sagan, Daua, Genale y Webi Shebeli) y otros seis lagos (Abe, Yardi, Beda, Koka, Ziway y Chew Bahir)( tablas 1 y 2; figuras 1 y 2).

\section{Discusión}

El cocodrilo del Nilo fue extremadamente abundante en los ríos y lagos de Etiopía hasta 1955, donde con el comienzo de la caza sistemática fue exterminado o reducido a densidades mínimas en muchas áreas (Whitaker \& Whitaker, 2007). No se ha realizado ningún estudio a gran escala que determine la distribución actual precisa ni las densidades por cuencas fluviales y masas lacustres. Tan sólo zonas concretas, y generalmente sometidas a explotación por caza deportiva, cuentan con planes de manejo y censos poblacionales como es el caso del lago Chamo (Shirley et al., 2014). En un país que presenta una amplia diversidad de hábitats idóneos para C. niloticus, con aproximadamente $70.000 \mathrm{~km}^{2}$ de áreas cubiertas por cuerpos de agua en forma de ríos, lagos y humedales asociados (Wood \& Talling, 1988), es paradójico que no se haya prestado más atención al mayor carnívoro de África.

Durante el trabajo de campo se ha observado una dilatada variabilidad en lo que a tiempo y esfuerzo de muestreo se precisa para detectar a la especie en una determinada zona húmeda. Factores como la densidad, presión cinegética sostenida y grado de cobertura vegetal ribereña resultan determinantes. En áreas de parques nacionales y reservas donde el reptil ha gozado legalmente de protección desde 1971, como son parte del curso superior del río Awash, sectores del río Omo y sector norte del lago Chamo (integrado dentro del Parque Nacional de Nech Sar), el asignar la presencia positiva de la especie requiere un mínimo esfuerzo de muestreo (figura 3). Por el contrario, espacios como los cauces 


\begin{tabular}{|c|c|c|c|c|}
\hline & DENOMINACION & LOCALIZACION & PRESENCIA & FUENTE \\
\hline 1 & MAREB & Frontera Eritrea & + & 3 \\
\hline 2 & TEKEZE & Frontera Eritrea & + & 3 \\
\hline 3 & ABBAI & Nilo Azul & + & 2,3 \\
\hline 4 & AWASH & Valle del Rift & + & $1,2,3$ \\
\hline 5 & GIBE & Valle del Rift & + & 1,3 \\
\hline 6 & GOJEBE & Valle del Rift & + & 1,3 \\
\hline 7 & $\mathrm{OMO}$ & Valle del Rift & + & $1,2,3$ \\
\hline 8 & BARO & Gambella & + & 2,3 \\
\hline 9 & ALUORO & Gambella & + & 2,3 \\
\hline 10 & GILO & Gambella & + & 2,3 \\
\hline 11 & AKOBO & Gambella & + & 2,3 \\
\hline 12 & HARE & Valle del Rift & + & 3 \\
\hline 13 & KULFO & Valle del Rift & + & $1,2,3$ \\
\hline 14 & SAGAN & Valle del Rift & + & 3 \\
\hline 15 & DAUA & Somali & + & 2,3 \\
\hline 16 & GENALE & Somali & + & 2,3 \\
\hline 17 & WEBI SHEBELI & Somali & + & 2,3 \\
\hline 18 & FAFEN SHET & Somali & - & 2 \\
\hline
\end{tabular}

Tabla 1.- Presencia ( + ) / Ausencia ( - ) de C. niloticus en ríos de Etiopía.

Fuente:

1.- Muestreos 2012.

2.- Comunicación EWCA.

3.- Información bibliográfica (Ayenew, 2009; Belay, 2009; Delleleng \& Nune, 2010; Derek 2010; Largen \& Spawls, 2010; Shirley et al., 2014; Tefera, 2014).

Table 1.- Presence ( + ) / Absence ( - ) of C. niloticus in rivers of Ethiopia.

Fuente:

1.- Samples 2012.

2.- Communication EWCA.

3.- Bibliographic information (Ayenew, 2009; Belay, 2009; Delleleng \& Nune, 2010; Derek 2010; Largen \& Spawls, 2010; Shirley et al., 2014; Tefera, 2014). 


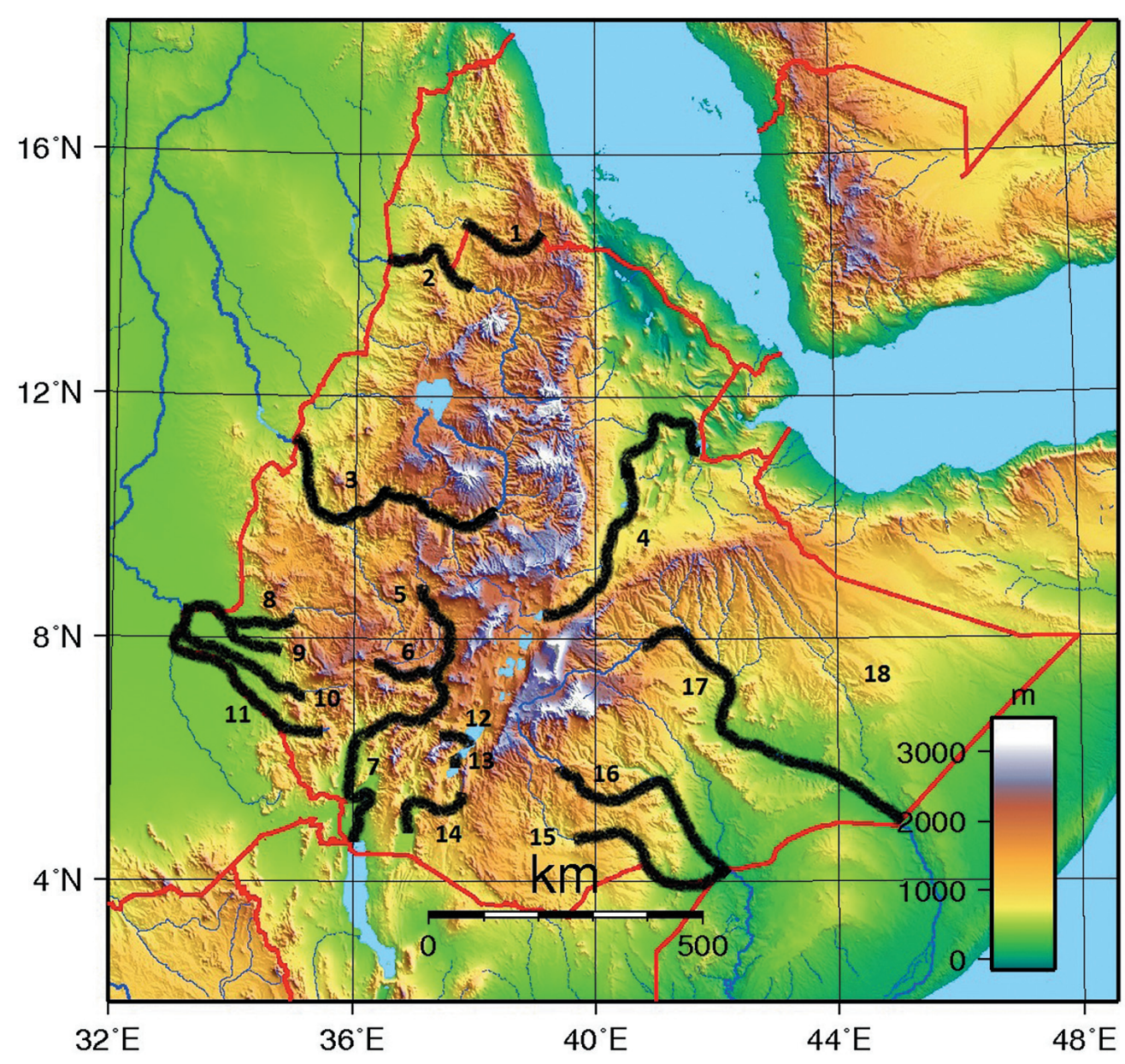

Fig. 1.- Distribución de C. niloticus (en negro) en ríos de Etiopía.
1.- Mareb
4.- Awash
7.- Omo
10.- Ghilo
13.- Kulfo
16.- Genale
2.- Tekeze
5.- Gibe
8.- Baro
11.- Akobo
14.- Sagan
17.- Webi Shebeli
3.- Abbai
6.- Gojebe
9.- Aluoro
12.- Hare
15.- Daua
18.- Fafem Shet

Fig. 1.- Distribution of C. niloticus (in black) in rivers of Ethiopia.
1.- Mareb
4.- Awash
7.- Omo
10.- Ghilo
13.- Kulfo
16.- Genale
2.- Tekeze
5.- Gibe
8.- Baro
11.- Akobo
14.- Sagan
17.- Webi Shebeli
3.- Abbai
6.- Gojebe
9.- Aluoro
12.- Hare
15.- Daua
18.- Fafem Shet 


\begin{tabular}{|ccccc|}
\hline & DENOMINACION & LOCALIZACION & PRESENCIA & FUENTE \\
\hline 1 & TANA & Tierras altas del noroeste & - & 1,3 \\
2 & ABE & Cuenca del Awash & + & 3 \\
3 & YARDI & Cuenca del Awash & + & 2,3 \\
4 & BEDA & Cuenca del Awash & + & 2,3 \\
5 & BESEKA & Cuenca del Awash & + & $1,2,3$ \\
6 & KOKA & Cuenca del Awash & + & 3 \\
7 & ZIWAY & Valle del Rift & + & 2,3 \\
8 & LANGANO & Valle del Rift & + & $1,2,3$ \\
9 & ABIYATA & Valle del Rift & - & $1,2,3$ \\
10 & SHALA & Valle del Rift & - & $1,2,3$ \\
11 & AWASA & Valle del Rift & - & 1,2 \\
12 & ABAYA & Valle del Rift & + & $1,2,3$ \\
13 & CHAMO & Valle del Rift & + & $1,2,3$ \\
14 & CHEW BAHIR & Valle del Rift & + & 3 \\
15 & TURKANA & Cuenca del Omo & + & $1,2,3$ \\
16 & MURLE & Cuenca del Omo & + & + \\
\hline
\end{tabular}

Tabla 2.- Presencia ( + ) / Ausencia ( - ) de C. niloticus en en lagos de Etiopía.

Fuente:

1.- Muestreos 2012.

2.- Comunicación EWCA.

3.- Información bibliográfica (Ayenew, 2009; Belay, 2009; Delleleng \& Nune, 2010; Derek 2010; Largen \& Spawls, 2010; Shirley et al., 2014; Tefera, 2014).

Table 2.- Presence ( + ) / Absence ( - ) of C. niloticus in lakes of Ethiopia.

Fuente:

1.- Samples 2012.

2.- Communication EWCA.

3.- Bibliographic information (Ayenew, 2009; Belay, 2009; Delleleng \& Nune, 2010; Derek 2010; Largen \& Spawls, 2010; Shirley et al., 2014; Tefera, 2014). 


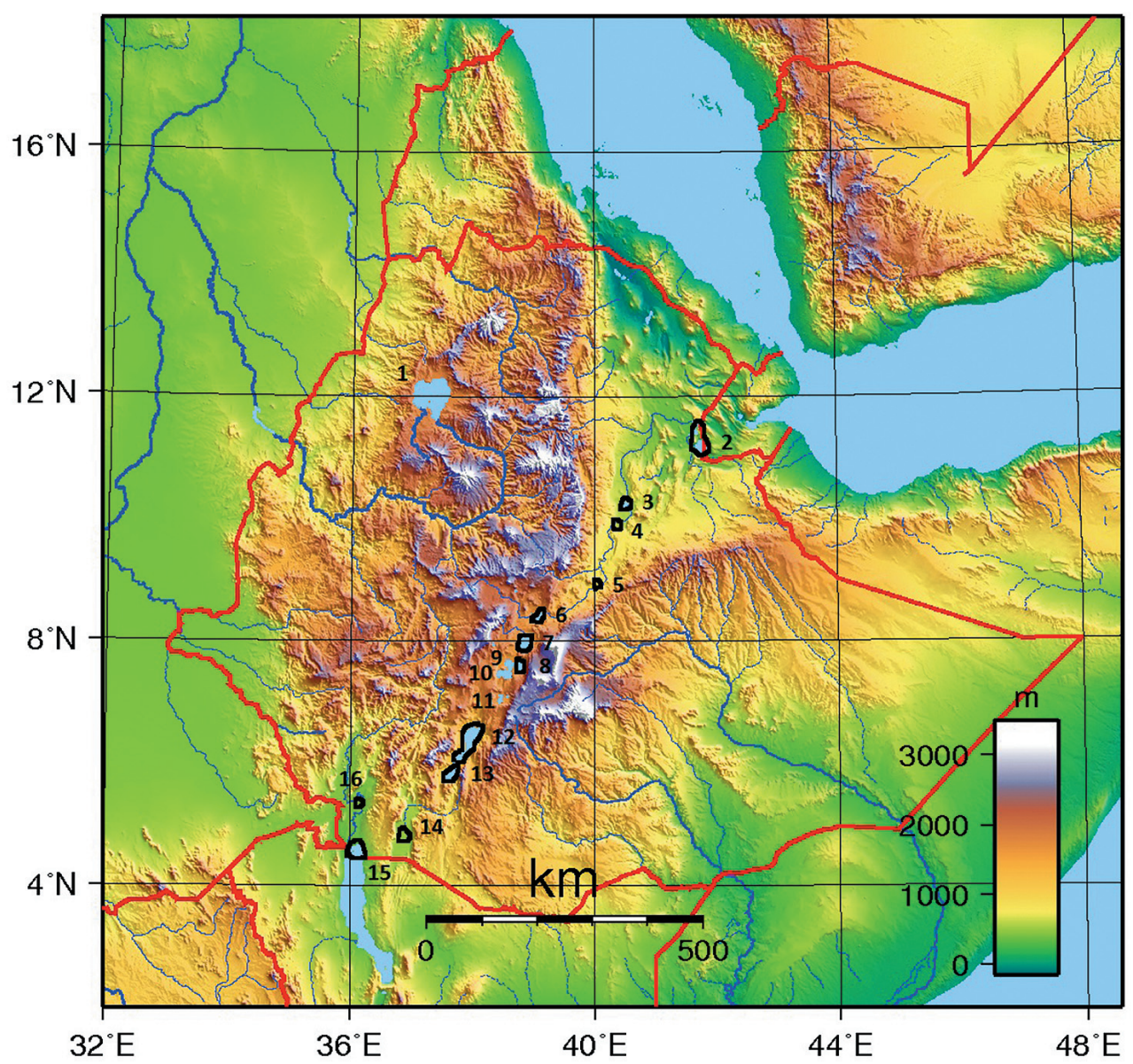

Fig. 2.- Distribución de C. niloticus (en negro) en lagos de Etiopía.
1.- Tana
5.- Beseka
9.- Abiyata
13.- Chamo
2.- Abe
6.- Koka
10.- Shala
14.- Chew Bahir
3.- Yardi
7.- Ziway,
11.- Awasa
15.- Turkana
4.- Beda
8.- Langano
12.- Abaya
16.- Murle

Fig. 2.- Distribution of C. niloticus (in black) in lakes of Ethiopia.
1.- Tana
5.- Beseka
9.- Abiyata
13.- Chamo
2.- Abe
6.- Koka
10.- Shala
14.- Chew Bahir
3.- Yardi
7.- Ziway,
11.- Awasa
15.- Turkana
4.- Beda
8.- Langano
12.- Abaya
16.- Murle 
fluviales de media entidad (5-7 m de anchura) con riberas densamente arboladas (río Kulfo) han requerido un mayor esfuerzo de muestreo. En perímetros lacustres con gran fluctuación del nivel hídrico la detección también es complicada, ya que no existen zonas predeterminadas de termorregulación o reproducción. Es especialmente ardua la tarea de localización de la especie en el sector etíope del lago Turkana, donde el nivel de las aguas ha descendido más de 10 m en las últimas décadas (Avery, 2010) y la principal fuente de alimentación de los cocodrilos, (tilapias Oreochromis niloticus, Linnaeus, 1758 y percas del Nilo Lates niloticus, Linnaeus, 1758) ha sido sobrexplotada (Kolding, 1993). En la actualidad los cocodrilos son perseguidos por los pescadores, que destruyen los lugares de nidificación incluso dentro de áreas protegidas como en el sector keniata del lago Turkana, (observ. pers. autor, 2002). La fluctuación del nivel del lago Beseka, situado en el Rift Valley dentro de la cuenca del río Awash, pero sin conexión directa con éste, también ha sido importante. Sin embargo, en esta zona húmeda el nivel ha subido considerablemente, hasta el punto de inundar carreteras y vías férreas adyacentes. El fenómeno es en este caso de origen geológico (tectónico-volcánico) y está siendo sometido a estudio y seguimiento (Belay, 2009). Aquí la población de cocodrilos es muy reducida y aislada, lo que demuestra la capacidad que tiene esta especie para desplazarse por tierra tramos significativos y colonizar cuerpos de agua comparativamente pequeños y alejados, como son los casos del lago Beseka y el oasis Filwoha (Largen \& Spawls, 2010). En cauces fluviales de entidad al norte del país, fronterizos con Eritrea, como los ríos Tekeze y Mareb, este reptil subsiste en un entorno muy árido. Una de las citas más antiguas de las que se dispone procede del río Mareb, donde Del Prato obtuvo registros de C. niloticus en 1891 (Largen, 1997). El entorno es muy similar a dos de los ríos de mayor entidad del cuadrante sudeste del país, dentro del distrito de Somali. En un medio semidesértico la especie se mantiene en los cauces de los ríos Genale y Webi Shebeli; sin embargo nunca ha sido citado en el río Fafem Shet (Largen \& Spawls 2010).

En medios lacustres integrados en la cuenca del Awash, la especie está presente en los lagos Koka (Tefera, 2014), Beda, Yardi (Largen \& Spawls, 2010) y Abe (Dellelegn \& Nune, 2010). En la mitad meridional del Valle del Rift etíope ocupa los lagos Langano, Abaya, Chamo (obs. pers.) y Chew Bahir (Largen \& Spawls, 2010).

Los vecinos lagos Abiyata y Shala se consideran demasiado alcalinos para albergar cocodrilos. El lago Awasa, de aguas dulces y abundantes recursos piscícolas, no ha ofrecido resultados positivos en las prospecciones (Figura 4). Durante el trabajo de campo se han recogido testimonios orales de la población local, buena conocedora del medio al vivir básicamente de la pesca. El argumento generalizado es que los cocodrilos han sido extinguidos en este lugar. Tampoco ha podido ser documentada su presencia a partir de fuentes bibliográficas ni por comunicaciones de miembros del EWCA.

El lago Ziway alberga las condiciones medioambientales idóneas para la especie: aguas dulces, riqueza piscícola con gran abundancia de tilapia, y altitud dentro del rango de tolerancia de C. niloticus. A pesar de ello no existen citas y las prospecciones realizadas en 2012 no ofrecieron resultados positivos. Sin embargo, existe el testimonio fotográfico de 


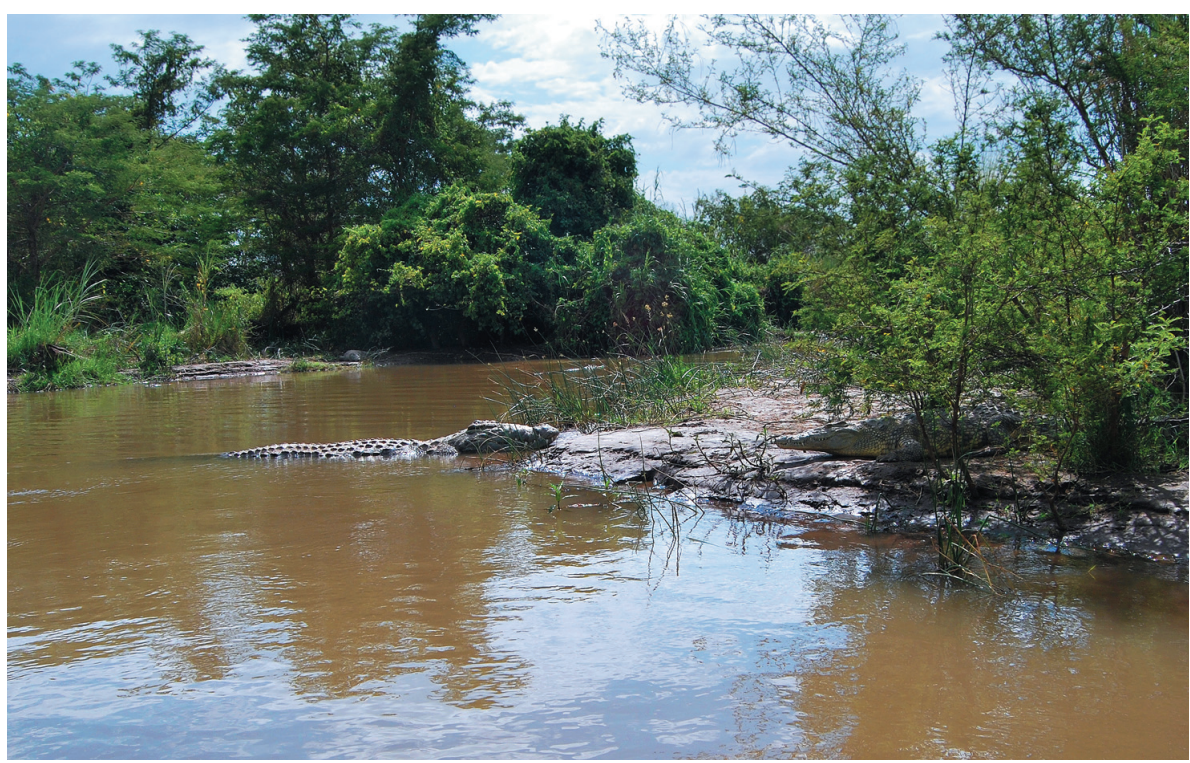

Fig. 3.- Área con alta densidad de C. niloticus. Desembocadura del río Kulfo al norte del Lago Chamo. Fig. 3.- High density area C. niloticus, Kulfo mouth of the river north of Lake Chamo.

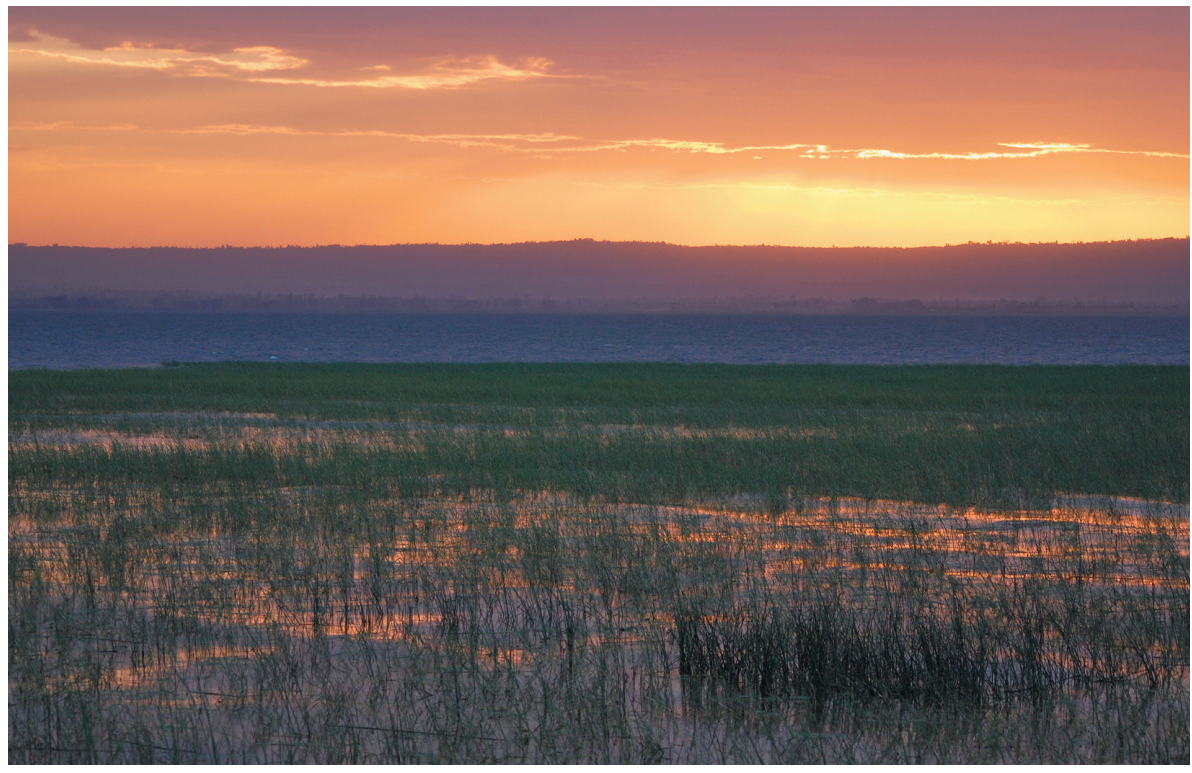

Fig. 4.- Área con hábitat óptimo y ausencia de registros de C. niloticus, Lago Awasa.

Fig. 4.- Area with optimum habitat and absence of records of C. niloticus, Lake Awasa. 
un adulto de tamaño medio termorregulando en área palustre a orillas del lago, fechado en 25.01.2014 y fotografiado en la Expedición Shoppe Ottawa por Jose Ponsoda, constituyendo la primera cita documentada para este lago.

El lago Tana, el mayor del país, ubicado en las tierras altas del noroeste a $1.840 \mathrm{~m}$ y origen del Nilo Azul, no alberga cocodrilos debido a su elevada altitud, tal y como apuntan varios investigadores (Nagelkerke, 1997; Largen \& Spawls, 2010). Históricamente no se han recogido citas de la existencia de cocodrilos en este gran lago. Es clarificador el testimonio de Leo Nagelkerke cuando escribe en su tesis doctoral: "Los cocodrilos están ausentes del lago Tana, pero están presentes río arriba desde las Cataratas Tissisat. Su situación no ha cambiado desde que Cheesman registró este hecho en 1936, y me pregunto por qué nunca han invadido el lago" (Nagelkerke, 1997).

\section{Lago Chamo: estimación de tamaño corporal para C. niloticus}

El lago Chamo, localizado al sur de Etiopia en la región de las Naciones, Nacionalidades y Pueblos del Sur, a una altitud de $1.110 \mathrm{~m}$ es conocido por albergar una de las mayores poblaciones de cocodrilos del continente, con ejemplares que alcanzan tallas descomunales. El mayor ejemplar registrado en el continente africano, con una longitud de 7,6 m corresponde a un gran macho cazado en marzo de 1903 por el alemán Hans Besser en el río Mbaka, tributario del lago Malawi (Siege, 2014). La literatura científica recoge especímenes que sobrepasaban los 5,5 m procedentes del río Semliki en Uganda, y de 5,3 m en el lago Rukwa (Tanzania) (Spawls et al., 2002).

\begin{tabular}{|cccccc|}
\hline $\begin{array}{c}\text { Cráneo } \\
\mathbf{N}^{\mathbf{0}}\end{array}$ & Ubicación & Procedencia & $\begin{array}{c}\text { LDC } \\
(\mathbf{c m})\end{array}$ & $\begin{array}{c}\text { MAC } \\
(\mathbf{c m})\end{array}$ & $\begin{array}{c}\text { LCT } \\
(\mathbf{m})\end{array}$ \\
\hline 1 & Nech Sar N. P. Museum & Lago Chamo & 68,6 & 18,5 & 5,35 \\
2 & Nech Sar N. P. Museum & Lago Chamo & 67,5 & 18,2 & 5,26 \\
3 & Arba Minch Crocodile Ranch & Lago Chamo & 65,5 & 17,7 & 5,11 \\
4 & Nech Sar N.P. Museum & Lago Chamo & 64 & 17,3 & 4,99 \\
5 & Arba Minch Crocodile Ranch & Lago Chamo & 62 & 16,7 & 4,84 \\
6 & Nech Sar N.P. Museum & Lago Chamo & 57,8 & 15,6 & 4,51 \\
7 & Nech Sar N.P.Museum & Lago Chamo & 53 & 14,3 & 4,13 \\
8 & Nech Sar N.P. Museum & Lago Chamo & 47,8 & 12,9 & 3,72 \\
\hline
\end{tabular}

Tabla 3.- Estimación de la longitud corporal total (LCT) de grandes ejemplares de C. niloticus procedentes del Lago Chamo, obtenida en función de la longitud dorsal (LDC) y la anchura máxima (MAC) de muestras craneales. Se indica la procedencia de dichas muestras.

Table 3.- Estimation of the total body length (LCT) of larges specimenes of C. niloticus from Lake Chamo, obtained according dorsal total lenght (LDC) and maximum width (MAC) of craneal samples. 

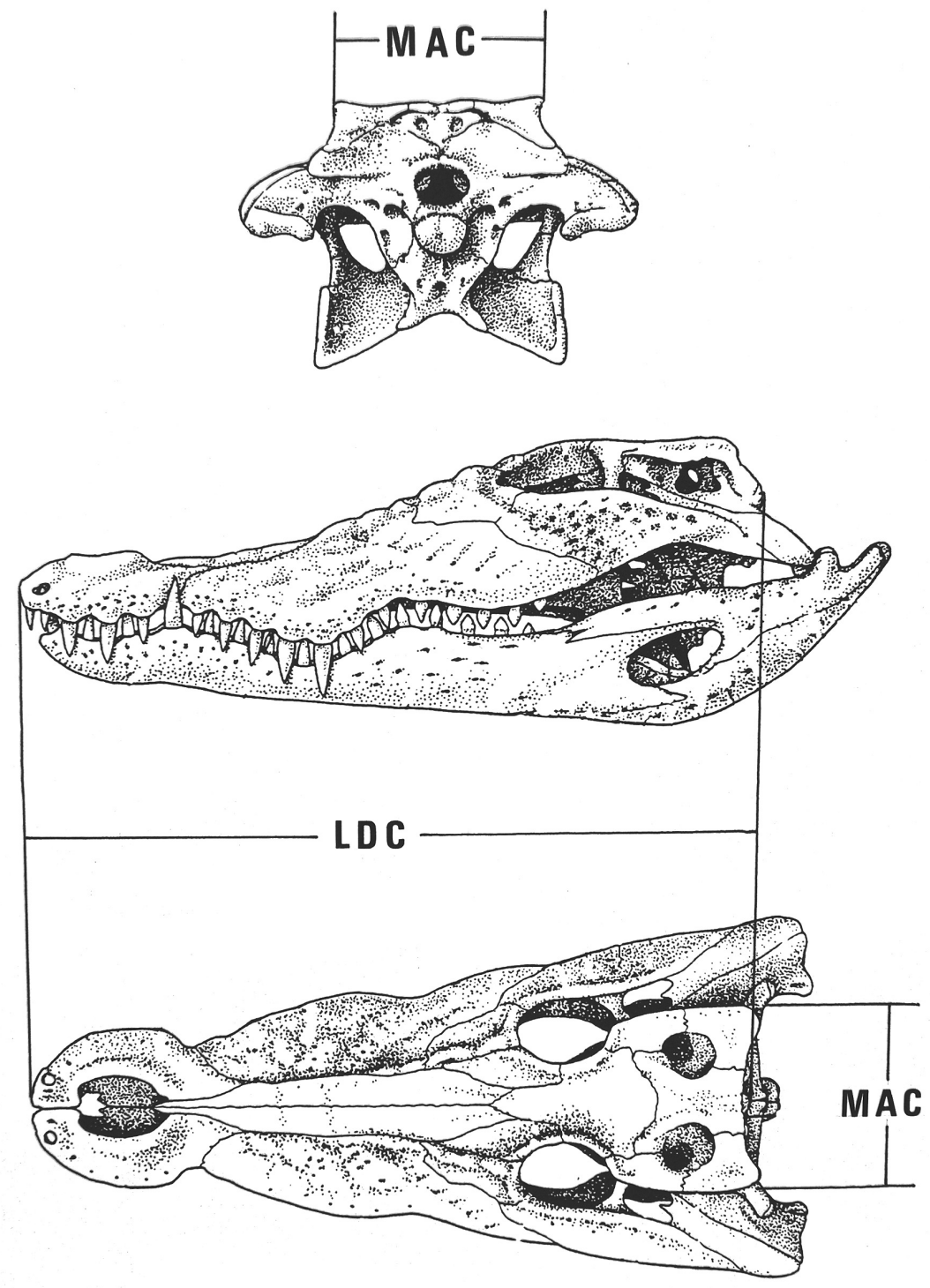

Fig. 5.- Cráneo de C. niloticus con señalización de las medidas estándar utilizadas: longitud dorsal craneal (LDC) y máxima anchura craneal (MAC).

Fig. 5.- Skull with signaling standard measures used: craneal total length (LDC) and maximum craneal width. 
En el lago Chamo, en censos llevados a cabo en 2004 se contabilizaron un total de 46 ejemplares que superaban los 5 m de longitud (Whitaker \&Whitaker, 2007). Estos mismos autores ofrecen estimaciones de tamaño corporal basadas en medidas craneales de especímenes procedentes del Chamo, que alcanzan los 5,4 m (Whitaker \& Whitaker, 2008). Recientemente se han citado capturas de individuos que alcanzan los 6,2 m (Derek, 2010). Se ha debatido que la genética peculiar de esta población sea el origen determinante de la existencia en gran número de individuos excepcionales por su tamaño, o bien que la estabilidad en el tiempo dentro de un espacio protegido, el Parque Nacional de Nech Sar, haya propiciado el desarrollo de animales muy longevos (Whitaker \&Whitaker, 2008). En el examen y posterior toma de datos biométricos de la selección de cráneos depositados en el Museo del Parque Nacional de Nech Sar y en el Centro de visitas de la Granja de cocodrilos de Arba Minch se obtuvieron estimas de tamaño corporal de tres ejemplares que superaban los $5 \mathrm{~m}$ de longitud. Otros dos ejemplares se acercaban a esa medida, con 4,99 m y 4,84 m de LCT (tabla 3; figura 5). No se logró ninguna muestra que superara los $6 \mathrm{~m}$, teniendo constancia, según testimonio verbal de miembros del EWCA, de la existencia de grandes machos territoriales que superan esa longitud en la orilla norte del lago.

La población de C. niloticus del lago Chamo es destacable por su peculiaridad y relevancia a nivel continental. El desplome de poblaciones estables y equilibradas en las últimas décadas, como la del Parque Nacional Murchison en Uganda y la del lago Turkana en Kenia (Avery 2010), incrementa el valor de esta población como una de las más representativas de África.

\section{Agradecimientos}

A Endale Moya y al resto de los miembros de la Ethiopian Wildlife Conservation Authority (EWCA), que me ofrecieron información y velaron por mi seguridad en los ríos y lagos etíopes. A Ayuba Ahmed por su gran competencia como rastreador en tierras africanas. A Abraham Abera excelente guía e intérprete sin cuya colaboración habría sido imposible la realización del estudio de campo. Al Ethiopian Mapping Authority (EMA) por poner a mi disposición cartografía especializada. Al personal de Nech Sar National Park Museum, Arba Minch Crocodile Ranch y Museo de Ciencias Naturales de Adis Abeba, que me permitieron acceder a sus fondos y me facilitaron información bibliográfica. A Brian Webster y Elena Potes por su contribución en la síntesis del informe.

\section{Bibliografía}

- Andeberhan, K. 1977. Lake Abaya and Lake Chamo Crocodile survive. Report to Government. Ethiopia. 
- Avery, S. 2010. Hydrological impacts of Ethiopia's Omo basin on Kenia's Lake Turkana water levels \& fisheries. African Development Bank Group. Nairobi.

- Ayenew, T. 2009. Natural Lakes of Ethiopia. Addis Ababa University Press. Addis Ababa.

- Belay, E. A. 2009. Growing lake with growing problems: integrate hydrogeological investigation on Lake Beseka, Ethiopia. Bonn.

- Bolton, M. 1973. Report on a survey of crocodile (C. niloticus) in Selected parts of Lake Margherita (Abaya). Report for Government. Addis Ababa.

- Chapman, C.M. 1969. The Nile Crocodile in Ethiopia. Unpublished. M.S. 18 pp.

- Chapman, C.M. 1973. Crocodiles. Proceedings of the Second Working Meeting of Crocodile Specialist Sponsored and arranged by the Survival Service and Ecology Comissions of IUCN. Ethiopia: 17-24. Morges (Switzerland).

- Dellelegn, Y., Nune, S. 2010. A Glimpse at Biodiversity Hotspots of Ethiopia. Mengistu Wondafrash (Ed.). EWNHS. Addis Ababa.

- Derek, L. 2010. An Introduction to the Natural History of Nech San National Park. Ethiopian Wildlife \& Natural History Society. Addis Ababa.

- Ferguson, R. 2010. Nile Crocodile (Crocodylus niloticus). States Survey and Conservation Action Plan. Crocodile Specialist Group. Darwin.

- Gebre, A.,Wakjira, K. 1996. Impact of Fishing methods on Crocodile of Lake Chamo. Crocodile Specialist Group. Newsletter 15: 5.

- Gebre, A. 2002. Crocodile Attack on the River. Crocodile Specialist Group. Newsletter 21: 4.

- Graham, A. 1968. The Lake RudolfCrocodile (Crocodylus niloticus) Population. University of East Africa. Nairobi.

- Hekkala, E., Shirley, M.H., Amato, G., Austin, J.D., Charter, S., Thorbjarnarson, J., Vliet, K.A., Houck's, M.L., Desalle, R., Blum, M.J. 2011. An ancient icon reveals new mysteries: mummy DNA resurrects a cryptic species within the Nile Crocodile. Mol. Ecol. 20: 4199-4215. Blackwell Publishing.

- IUCN. 1973. Crocodile. IUCN Publication new series. Supplementary Paper 41. Switzerland.

- IUCN.1982. Crocodiles. IUCN Publication new series. Gainesville, Florida.

- Kolding, J. 1993. Population dynamics and life historystyles of Nile Tilapia, Oreochromis niloticus, in Ferguson's Gulf, Lake Turkana, Kenya. Environ. Biol. Fishes 37: 25-46.

- Lainez, D. 2009. Nile Crocodile survey database. WCMC. Cambridge.

- Largen, M. 1997. An annotated checklist of the amphibians and reptiles of Eritrea, with keys for their identification. Tropical Zool. 10 (1): 63-115.

- Largen, M., Spawls, S. 2010. The Amphibians and Reptiles of Ethiopia and Eritrea. Chimaira. Frankfurt am Main.

- Nagelkerke, L. 1997. The Barbs of Lake Tana, Ethiopia: morphological diversity and its implications for taxonomy, trophic resource partitioning, and fisheries. Doctoral thesis. AH Wageningen. Netherland. 
- Shirley, M.H., Oduro, W., Yaokokore-Beibro, H. 2009. Conservation status of crocodile in Ghana and Côte-d'Ivoire, West Africa. Oryx 43: 136-145.

- Shirley, M.H., Siege, L., Ademasu, M. 2014. Crocodile Management in Ethiopia. Crocodile Specialist Group. Darwin.

- Siege, L. 2014. The Monster Crocs of Lake Chamo. African Indaba 12 (4). Hungary.

- Spawls, S., HowelL, K., Drewes, R., Ashe, J. 2002. A Field Guide to the Reptiles of East Africa. Academic Press. Hong Kong.

- Tefera, M. 2014. Status of Nile Crocodile (Crocodylus niloticus) in Ethiopia and its Role as Biometrology Indicator. Addis Ababa.

- Webb, G.J.W., Smith, A.M.A. 1987. Life history parameters, populations dynamics and the management of crocodilians. In: Wildlife Management: Crocodiles and Alligators. G.I.W. Webb, S.C.Manolis, and P.J. Whitehead (Eds.): 199-210. Surrey Beatty and Sons. Sydney.

-Whitaker, R. 2007. The Nile Crocodile of Lake Chamo. Crocodile Specialist Group 26 (2). Darwin.

- Whitaker, R., Whitaker, N. 2007. Nech Sar National Park Proyect. Sustainable Use of the Lake Chamo Nile Crocodile Population. African Parks (Ethiopia). Addis Ababa.

-Whitaker, R., Whitaker, N. 2008. Who's got the biggest? Crocodile Specialist Group. Newsletter 27: 26-30. Darwin.

- Wood, R.B., Talling, J.F. 1988. Chemical and Algal Relationship in a salinity series of Ethiopian inland waters. Hydrobiologica 158: 29-67

\section{gै}

- Fecha de recepción/Date of reception: 29-07-2015

- Fecha de aceptación/Date of acceptance: 06-10-2015

Editor Asociado/Associate editor: Alberto Gosá 